IMSc - $92-17(\mathrm{Rev})$

\title{
Asymptotic Analysis and Spectrum of Three Anyons
}

\author{
G.Date, M.Krishna and M.V.N.Murthy \\ The Institute of Mathematical Sciences, Madras 600 113, India
}

\begin{abstract}
The spectrum of anyons confined in harmonic oscillator potential shows both linear and nonlinear dependence on the statistical parameter. While the existence of exact linear solutions have been shown analytically, the nonlinear dependence has been arrived at by numerical and/or perturbative methods. We develop a method which shows the possibility of nonlinearly interpolating spectrum. To be specific we analyse the eigenvalue equation in various asymptotic regions for the three anyon problem.
\end{abstract}




\section{Introduction}

Non-relativistic quantum mechanics in two spatial dimensions admits possibility of fractional statistics [1, 2]. Existence of fractional statistics is also intimately tied to having multivalued wave functions which occur naturally in the quantum mechanics on multiply connected spaces [3]. The classification of multivalued wave functions is provided by one dimensional representations of the fundamental group of the multiply connected configuration space. Although there are several known examples of a kinematic classification [4] (i.e., representations of the fundamental group), very little is known about the dynamics on such spaces. A system of "Anyons"- particles in two dimensions obeying fractional statistics- is a good and physically relevent example of quantum mechanics on multiply connected spaces. It is physically relevent because anyons have been proposed as candidates for explaining fractional quantum Hall effect [5] and are likely candidates for high $T_{c}$ superconduc-

tivity [6]. It is a good example because at least some exact solutions to the energy eigenvalue problem for $\mathrm{N}$-anyons in some external confining potential are known - an exception in many-body quantum mechanics with non-seperable Hamiltonians.

Concentrating on anyons in a harmonic oscillator potential, one notices two important features: All the exactly known energy eigenvalues are linear in the statistical parameter $\alpha$ for arbitrary number of anyons [7]. Whereas in the case of three and four anyons, numerical studies exhibit some eigenvalues with non-linear dependence on $\alpha$ as well as level crossings [\&]. Meanfield studies for large number of anyons also exhibit the same feature [9]. There 
is no analytical approach exhibiting such nonlinearly interpolating energy eigenvalues- raising perhaps a doubt that the nonlinearity may be an artifact of numerical computations which are necessarily limited by computing power. In the present work we develop an analytical approach which does show the possibility of nonlinearly interpolating spectrum. We believe that the method is general enough to have validity even beyond the domain of the problem on hand.

Our approach is based on analysing the eigenvalue equation (differential equation) in various asymptotic regions. Noting that eigenvalues themselves are independent of where the differential equation is solved, we expect the asymptotic form of the differential equation to determine the spectrum. Presently we have analysed in detail the case of three anyons in an oscillator potential but we believe the method can be suitably adapted to many anyons and possibly to some other many body systems.

The three anyon problem in the asymptotic regions we consider reduces to solving a Confluent Hypergeometric Equation (CHE) in the region $R \leq$ $x<\infty$. This equation has a solution regular at $x=0$ and a solution which is irregular at $x=0$. The regular solution leads to linearly interpolating energies while the irregular one ( $\mathrm{R}$ is strictly greater than zero) leads to nonlinearly interpolating solutions. We give two first order equations which specify the spectrum exactly.

The paper is organised as follows: In Sec.2 we discuss the preliminaries, specify our formulation and set the notation. This part is well known and has been included to make the paper self contained. In Sec.3, we give a quick review of known exact solutions. We point out how nonlinearity can come 
about and note additional asymptotic regions available to extract information about the spectrum. After specifying the asymptotics we derive the equations determining the spectrum. We note how the numerically known low lying eigenvalues fit into our equations. Full analysis of these system of equations is under investigation. Section 5 contains summary and a brief discussion.

\section{Preliminaries}

In the following by "anyons" we mean a quantum mechanical system of $\mathrm{N}$ particles moving in two dimensions with wave functions which have a stipulated multi-valuedness. To make this explicit let us denote a generic wave function as $\psi\left(\vec{r}_{1}, \ldots, \vec{r}_{N}\right)$. Let $P_{i j \gamma}$ denote the operation of taking the ith particle coordinate around the jth coordinate along a closed path $\gamma$. The path $\gamma$ does not enclose any other particle coordinate and is taken in an anticlockwise sense, say. Then under such an operation $\psi$ acquires a phase namely,

$$
P_{i j \gamma} \psi\left(\vec{r}_{1}, \ldots \vec{r}_{N}\right)=\exp (i 2 \pi \alpha) \psi\left(\vec{r}_{1}, \ldots \vec{r}_{N}\right)
$$

If a path $\gamma$ encloses other particle coordinates as well then such a path can be broken into a set of closed paths each of which encloses exactly one particle coordinate. Applying the stipulation above, one can compute the total phase, for such a path. If the sense of the path is reversed then $\alpha \rightarrow-\alpha$. Clearly the phase acquired depends only on the homotopy class of the path (i.e., is same for two paths $\gamma$ and $\gamma^{\prime}$ if $\gamma$ and $\gamma^{\prime}$ can be continuously deformed into each other). 
Let us introduce the complex notation for particle coordinates: $Z_{j}=$ $x_{j}+i y_{j}, \bar{Z}_{j}=\bar{x}_{j}-i \bar{y}_{j}$ coordinates. Clearly $Z_{i j}$, where $Z_{i j}=Z_{i}-Z_{j}$, has the property that if $Z_{j}$ is taken around $Z_{i}, Z_{i j}^{\alpha}$ changes by $\exp (i 2 \pi \alpha)$. This allows us to write any generic wave function satisfying eqn(1) as,

$$
\psi\left(Z_{i}, \bar{Z}_{i}\right)=\left[\prod_{i<j}\left(\frac{Z_{i j}}{\bar{Z}_{i j}}\right)^{\alpha / 2}\right] \tilde{\psi}\left(Z_{i}, \bar{Z}_{i}\right)
$$

with the bracketed expression being a phase and now $\tilde{\psi}\left(Z_{i}, \bar{Z}_{i}\right)$ is a single valued function.

Clearly,

$$
\nabla_{k} \psi\left(Z_{i}, \bar{Z}_{i}\right)=\prod_{i<j}\left(\frac{Z_{i j}}{\bar{Z}_{i j}}\right)^{\alpha / 2}\left[\nabla_{k} \tilde{\psi}\left(Z_{i}, \bar{Z}_{i}\right)+\nabla_{k} \ln \left(\prod_{i<j}\left(\frac{Z_{i j}}{\bar{Z}_{i j}}\right)^{\alpha / 2}\right) \tilde{\psi}\left(Z_{i}, \bar{Z}_{i}\right)\right] .
$$

which can be rewritten as,

$$
\nabla_{k} \psi\left(Z_{i}, \bar{Z}_{i}\right)=\prod_{i<j}\left(\frac{Z_{i j}}{\bar{Z}_{i j}}\right)^{\alpha / 2}\left[\nabla_{k} \tilde{\psi}\left(Z_{i}, \bar{Z}_{i}\right)+i \alpha \sum_{j \neq k} \frac{\hat{z} \times \vec{r}_{k j}}{\left|\vec{r}_{k j}\right|^{2}} \tilde{\psi}\left(Z_{i}, \bar{Z}_{i}\right)\right] .
$$

Since $\tilde{\psi}$ is single valued, the r.h.s of the above equation has exactly the same multivaluedness as the l.h.s. In otherwords we have,

$$
\nabla_{k}\left[\prod_{i<j}\left(\frac{Z_{i j}}{\bar{Z}_{i j}}\right)^{\alpha / 2} \tilde{\psi}\left(Z_{i}, \bar{Z}_{i}\right)\right]=\prod_{i<j}\left(\frac{Z_{i j}}{\bar{Z}_{i j}}\right)^{\frac{\alpha}{2}} D_{k} \tilde{\psi}
$$

where

$$
D_{k} \tilde{\psi}=\left[\nabla_{k}+\vec{A}_{k}\right] \tilde{\psi}
$$

and

$$
A_{k}\left(\vec{r}_{k}\right)=i \alpha \sum_{j \neq k} \frac{\hat{z} \times \vec{r}_{k j}}{\left|\vec{r}_{k j}\right|^{2}}
$$

This allows us to write any higher order differential operators on $\psi$ in terms of corresponding covariant differential operators on the singlevalued 
wave function $\tilde{\psi}$. In particular a Hamiltonian operator, typically $-\sum_{i} \nabla_{i}^{2}+V$ can be written similarly. An eigenvalue equation written in terms of $\psi$ can then be recast as a corresponding equation in terms of $\tilde{\psi}$.

Although both formulations are equivalent, dealing with operators on multivalued wave functions is much less transparent than dealing with operators on singlevalued wave functions. Naive commutation rules, symmetries that one would expect by looking at an operator on single valued functions are not at all true in general for the "same" differential operators acting on multivalued wave function.

Considering eigenvalue problem in terms of $\tilde{\psi}$ has other advantages too. Since all the subtleties of multivaluedness are equivalently transcribed in terms of additional "interaction" terms (the so called statistical interactions), the eigenvalue problem is amenable to approximations. One is also on firmer ground in doing usual algebraic manipulations with operators. With these in mind we will work with singlevalued wave functions with "statistical interactions".

As a first step one would like to understand the system of "free anyons". However, the statistical interaction falls off as $\left|r_{i j}\right|^{-2}$ as $\left|r_{i j}\right| \rightarrow \infty$. So one is not sure whether the Hamiltonian with only statistical interactionss has only discrete eigenvalues. One can put the system in a box to ensure discrete eigenvalues but then one needs suitable boundary condition. An oscillator potential ensures discrete spectrum without introducing a finite size. One could take some other confining potential but in the limit $\alpha \rightarrow 0$ one should know the spectrum. One then has hope of doing at least the perturbative analysis. Since the statistical interaction depends only on rel- 
ative separations, the Centre of $\operatorname{Mass}(\mathrm{CM})$ dynamics should play a trivial role and oscillator potential also allows a separation of CM and relative coordinate dynamics. The oscillator potential problem can also be mapped on to a problem of anyons in a real, constant external magnetic field along the Z-axis [10]. Bearing these facts in mind, we choose the oscillator potential without further justification. In order to derive the thermodynamic properties of a system of anyons there exist well defined methods of eliminating the dependence on the oscillator frequency [11].

The Hamiltonian we consider is- after carrying out the usual scaling of variables $(\hbar=c=1)$ - interms of dimensionless quantities,

$$
H=\hbar \omega\left[\frac{1}{2} \sum_{i=1}^{N} p_{i}^{2}+\frac{1}{2} \sum_{i=1}^{N} r_{i}^{2}-\alpha \sum_{j>i=1}^{N} \frac{\ell_{i j}}{r_{i j}^{2}}+\frac{\alpha^{2}}{2} \sum_{i \neq j, k}^{N} \frac{\vec{r}_{i j} \cdot \vec{r}_{i k}}{r_{i j}^{2} r_{i k}^{2}}\right]
$$

where

$$
\ell_{i j}=\left(\vec{r}_{i}-\vec{r}_{j}\right) \times\left(\vec{p}_{i}-\vec{p}_{j}\right)
$$

and all distances have been expressed in units of $1 / \sqrt{m \omega}$. Notice that the statistical interaction is independent of the centre of mass. This is the operator we analyse subsequently.

\section{Asymptotic Analysis}

First, we briefly discuss the class of exact solutions already known and then move over to the asymptotic analysis. For discussing the known class of exact

solutions it is convenient to use the complex coordinates $Z_{i}, \bar{Z}_{i}$ in terms of 
which the Hamiltonian takes the form,

$$
H=-2 \partial_{i} \bar{\partial}_{i}+\frac{1}{2} Z_{i} \bar{Z}_{i}-\alpha \sum_{i<j}\left(\frac{\partial_{i j}}{\bar{Z}_{i j}}-\frac{\bar{\partial}_{i j}}{Z_{i j}}\right)+\frac{\alpha^{2}}{2} \sum_{i \neq j, k} \frac{1}{\bar{Z}_{i j} Z_{i k}}
$$

where $\partial_{i}=\partial / \partial Z_{i} ; \partial_{i j}=\partial_{i}-\partial_{j}$, etc., and the eigenvalue equation is,

$$
H \psi\left(Z_{i}, \bar{Z}_{i}\right)=E \psi\left(Z_{i}, \bar{Z}_{i}\right)
$$

The conserved angular momentum J, is given by,

$$
J=Z_{i} \partial_{i}-\bar{Z}_{i} \bar{\partial}_{i}
$$

For the sake of completeness we recapitulate the standard asymptotic analysis, i.e, $Z_{i}=\lambda \hat{Z}_{i}, \lambda \rightarrow \infty$ :

(i) As $Z_{i} \rightarrow \infty, Z_{i j} \rightarrow \infty$, the oscillator potential term dominates over the $\alpha$ dependent terms reducing $H$ to the Hamiltonian of $\mathrm{N}$ two dimensional oscillators. All the eigenfunctions of the oscillator have the Gaussian suppression factor. Thus we put,

$$
\psi\left(Z_{i}, \bar{Z}_{i}\right)=\exp \left(-\frac{1}{2} \sum_{i} Z_{i} \bar{Z}_{i}\right) \psi_{1}\left(Z_{i}, \bar{Z}_{i}\right)
$$

Substitution of this form transforms the original eigenvalue equation to an equation for $\psi_{1}$, viz.,

$$
\left[-2 \partial_{i} \bar{\partial}_{i}+Z_{i} \partial_{i}+\bar{Z}_{i} \bar{\partial}_{i}-\alpha \sum_{i<j}\left(\frac{\partial_{i j}}{\bar{Z}_{i j}}-\frac{\bar{\partial}_{i j}}{Z_{i j}}\right)+\frac{\alpha^{2}}{2} \sum_{i \neq j, k} \frac{1}{\bar{Z}_{i j} Z_{i k}}\right] \psi_{1}=(E-N) \psi_{1}
$$

The $\mathrm{N}$ on the r.h.s is the zero point energy for $\mathrm{N}$ two dimensional oscillators while on the l.h.s the oscillator potential is traded for the "scaling operator", $Z_{i} \partial_{i}+\bar{Z}_{i} \bar{\partial}_{i}$. 
(ii) The other asymptotic region is $\left|Z_{i j}\right| \rightarrow 0$ for any pair of particles $\mathrm{i}$ and j, say. Since the $\alpha$ dependent terms are singular we expect the wave function to vanish sufficiently fast in this limit so that the eigenvalue equation is well defined.

Assuming a Taylor series expansion in $Z_{i j}$ as $\left|Z_{i j}\right| \rightarrow 0$, we can write,

$$
\psi_{1}\left(Z_{i}, \bar{Z}_{i}\right) \longrightarrow \sum_{a \geq 0}\left(\left|Z_{12}\right|\right)^{\lambda+a} \psi_{1 a}\left(Z_{2}, \ldots, \bar{Z}_{2}, \ldots\right)
$$

for $\lambda \geq 0$ and we have taken the pair (12) for definiteness. Substituting this in the eigenvalue equation and keeping terms to the leading order in $\left|Z_{12}\right|$ gives,

$$
\lambda^{2}-\alpha^{2}=0
$$

or $\lambda= \pm \alpha$. However $\lambda>0$, thus $\lambda=\alpha$. Since this must happen for each pair of particles seperately and independently we can take out a factor $|X|^{\alpha}$, where

$$
X=\prod_{i<j} Z_{i j}
$$

For future reference let us also note that if

$$
\psi_{1}=X^{\beta / 2} \bar{X}^{\gamma / 2} \psi_{2}
$$

then the eigenvalue equation transforms to

$$
\begin{aligned}
& {\left[-2 \partial_{i} \bar{\partial}_{i}+Z_{i} \partial_{i}+\bar{Z}_{i} \bar{\partial}_{i}-(\alpha+\gamma) \sum_{i<j} \frac{\partial_{i j}}{\bar{Z}_{i j}}+(\alpha-\beta) \sum_{i<j} \frac{\bar{\partial}_{i j}}{Z_{i j}}+\right.} \\
& \left.\frac{(\alpha-\beta)(\alpha+\gamma)}{2} \sum_{i \neq j, k} \frac{1}{\bar{Z}_{i j} Z_{i k}}\right] \psi_{2}=\left[E-N-\frac{(\beta+\gamma)}{4} N(N-1)\right] \psi_{2}
\end{aligned}
$$

Taking $\beta=\gamma=\alpha$, we have,

$$
\psi_{1}=|X|^{\alpha} \psi_{2}
$$


and

$$
\left[-2 \partial_{i} \bar{\partial}_{i}+Z_{i} \partial_{i}+\bar{Z}_{i} \bar{\partial}_{i}-2 \alpha \sum_{i<j} \frac{\partial_{i j}}{\bar{Z}_{i j}}\right] \psi_{2}=\left[E-N-\frac{\alpha}{2} N(N-1)\right] \psi_{2}
$$

while if $\beta=\gamma=-\alpha$, we have,

$$
\psi_{1}=|X|^{-\alpha} \psi_{2}^{\prime}
$$

and

$$
\left[-2 \partial_{i} \bar{\partial}_{i}+Z_{i} \partial_{i}+\bar{Z}_{i} \bar{\partial}_{i}+2 \alpha \sum_{i<j} \frac{\bar{\partial}_{i j}}{Z_{i j}}\right] \psi_{2}^{\prime}=\left[E-N+\frac{\alpha}{2} N(N-1)\right] \psi_{2}^{\prime}
$$

Clearly in the second case $\psi_{2}^{\prime}$ will have to vanish at least as fast as $|X|^{2 \alpha}$.

Also, if $J \psi_{1}=j_{1} \psi_{1}$, then for general $\beta, \gamma$ we have

$$
J \psi_{2}=\left[j_{1}-\frac{N(N-1)}{2} \frac{\beta-\gamma}{2}\right] \psi_{2}=j_{2} \psi_{2} .
$$

For the two cases noted above, $\beta=\gamma= \pm \alpha, j_{2}=j_{1}$. Incidentally if $\beta=$ $\alpha, \gamma=-\alpha$ choice is made, then

$$
\psi_{1}=\left(\frac{X}{\bar{X}}\right)^{\alpha / 2} \psi_{2}^{\prime \prime}
$$

and $\psi_{2}^{\prime \prime}$ satisfies the simple equation,

$$
\left[-2 \partial_{i} \bar{\partial}_{i}+Z_{i} \partial_{i}+\bar{Z}_{i} \bar{\partial}_{i}\right] \psi_{2}^{\prime \prime}=[E-N] \psi_{2}^{\prime \prime}
$$

That is we recover the so called "anyon gauge" where the wave functions are multivalued. If we want to retain the singlevaluedness of the wave function then our choices are limited to $\gamma=\beta+$ integer. The three-body interaction term vanishes if $\gamma=-\alpha \operatorname{or} \beta=\alpha$. Allowing a non-zero integer only changes 
the angular momentum of the wave function with a proportionate change in the energy. We will, to begin with retain the angular momentum also and thus have $\beta=\gamma= \pm \alpha$ as the two choices.

At this stage we can summarise the known exact solution in a convenient way [7]. With the choice $\beta=\gamma=\alpha, \psi_{1}=|X|{ }^{\alpha} \psi_{2}$, we have two basics types of exact solutions:

$$
\psi_{2}=\psi_{2}\left(\bar{Z}_{i}\right) ; \quad j<0
$$

with the energy eigenvalues given by,

$$
E=N-j+\alpha \frac{N(N-1)}{2}
$$

and

$$
\psi_{2}=\psi_{2}\left(t=\sum_{i}\left|Z_{i}\right|^{2}\right) ; \quad j=0 .
$$

with $\psi_{2}(t)$ a polynomial of degree $\mathrm{m}$ in $\mathrm{t}$. The corresponding energy eigenvalues are given by,

$$
E=N+2 m+\alpha \frac{N(N-1)}{2},
$$

The second solution is necessarily bosonic since $t$ is symmetric, where as the first solution needs explicit symmetrization and antisymmetrization of the wavefunction in terms of $\bar{Z}_{i}$ to obtain the bosonic and fermionic wavefunctions. Since this is always possible, the degeneracy of the first type solution is exactly the same for both bosonic and fermionic type solutions for any given angular momentum $\mathrm{j}(<0)$; 
Obtaining the first solution is trivial since only the "scaling operator" contributes and $-j$ in the energy eigenvalue is nothing but the degree of scaling. The second solution can be obtained most easily by transforming the equation in the form of a confluent hypergeometric equation:

$$
\left[t \frac{d^{2}}{d t^{2}}+(b-t) \frac{d}{d t}-a\right] \psi_{2}(t)=0
$$

where

$$
\begin{gathered}
a=-\frac{1}{2}\left[E-N-\alpha \frac{N(N-1)}{2}\right] \\
b=N+\alpha \frac{N(N-1)}{2}
\end{gathered}
$$

The admissible solutions are the regular Confluent Hypergeometric Functions(CHF), $M(a, b, t)$. That $a=-m$ follows from the demand of normalizability of the wave function. The corresponding eigenvalues follow immediately.

We may also take a combination (product) of the solutions the types discussed above, Eqns(21) and (23), to get further $j<0$ solutions. These are :

$$
\psi_{2}=f\left(\bar{Z}_{i}\right) g(t) ; \quad E_{m, j}=2 m-j+N+\alpha \frac{N(N-1)}{2} .
$$

- With the choice $\beta=\gamma=-\alpha$ and $\psi_{1}=|X|^{-\alpha} \psi_{2}$ we have only the analouge of the first type of solutions i.e. $\psi_{2}=\psi_{2}\left(Z_{i}\right) . \quad \psi_{2}$ has to be of the form $X^{d} f\left(Z_{i}\right)$ with $d \geq 2 \alpha$ so that $\psi_{1}$ vanishes if the coordinates of any two particles coincide. This means that $\mathrm{j}$ has to be $\operatorname{large} \operatorname{enough}(j>(N-1)(N-2) / 2)$. The corresponding eigenvalues 
are given by

$$
E_{j}=j+N-\alpha \frac{N(N-1)}{2} .
$$

If however the condition, $j>(N-1)(N-2) / 2$, is not satisfied then the wave function $\psi_{1}$ remains regular only for some values of $\alpha$ (0 $\leq$ $\alpha \leq 2 j / N(N-1))$ but not for all $0 \leq \alpha \leq 1$ which gives rise to the so called noninterpolating solutions which have also been discussed in the literature 13. To the best of our knowledge these are all the exactly known solutions for the $\mathrm{N}$ anyon problem.

All these solutions for the energy eigenvalues have a linear dependence on $\alpha$ while the corresponding eigenfunctions are finite order polynomials apart from the $|X|^{\alpha}$ and the Gaussian factor. In fact a simple scaling arguement shows that if an exact eigenfunction is a polynomial (i.e. has a finite degree), then the corresponding eigenvalue must be linear in $\alpha$.

For, if $\psi_{2}$ is a polynomial in $Z_{i}, \bar{Z}_{i}$, with highest total degree $\mathrm{d}$ then for $Z_{i}, \bar{Z}_{i} \rightarrow \infty$ the polynomial becomes a monomial and only the scaling operator term dominates giving,

$$
E=N+d+\alpha \frac{N(N-1)}{2}
$$

Now if $\left|Z_{i j}\right| \rightarrow 0$ every eigenfunction vanishes as $\left|Z_{i j}\right|^{\alpha}$ or faster, i.e., after $|X|^{\alpha}$ is taken out the remaining function has only integer powers of $Z_{i}, \bar{Z}_{i}$. Thus d has to be an integer and hence E has a specific linear dependence on $\alpha$.

However it is well known that solutions which have nonlinear dependence exist. This has been shown numerically [8], through perturbative analysis 
[12] for three anyon problem and using meanfield methods for large number anyons [9]. This point is also our focus from now on. The scaling arguement will then imply that $\mathrm{d}$ has to be a nonlinear function of $\alpha$. How is this achieved?

Suppose $\psi_{2}$ admits a power series representation with infinite radius of convergence but the series does not truncate then the scaling arguement fails. However the analysis of such a series solution always seems to lead to exponentially divergent behaviour making the solution non-normalizable, ie., the power series has to truncate. But then only linear soutions are possible. The other possibility then is that the power series has a finite radius of convergence, i.e., a "scale" $\mathrm{R}$ has to enter if nonlinearity is to be possible. But this means that $\psi_{2}$ has two different representations as the scale parameter $\lambda \rightarrow 0$ and $\lambda \rightarrow \infty$, each being valid for $\lambda<\lambda_{\max }$ and $\lambda>\lambda_{\min }$ respectively. For $\lambda \geq \lambda_{\min }$ the scaling arguement can still work but now the series need not have only integer powers, i.e., $\mathrm{d}$ can be a nonlinear function of $\alpha$. We conclude then that if a nonlinearly interpolating eigenvalue is possible at all, the corresponding eigenfunction must have two different series representations for $\psi_{2}\left(\lambda \hat{Z}_{i}, \lambda \hat{\bar{Z}}_{i}\right)$ as $\lambda \rightarrow 0$ and $\lambda \rightarrow \infty$. One then has to try to match the two series suitably.

Exploring this possibility looks extremely complicated for general N. So we now specialise to $\mathrm{N}=3$ and go over to relative coordinate dynamics to reduce the number of independent variables in the differential equation. These are given interms of the Jacobi coordinates,

$$
\rho_{1}=\frac{Z_{1}-Z_{2}}{\sqrt{2}}
$$




$$
\rho_{2}=\frac{Z_{1}+Z_{2}-2 Z_{3}}{\sqrt{6}}
$$

and similarly their complex conjugates.

After separating out the CM coordinate and using the notation $\rho_{i}=$ $r_{i} \exp \left(i \theta_{i}\right)$ the relevant hamiltonion becomes,

$$
\begin{aligned}
H_{r e l}= & -\frac{1}{2} \sum_{i=1,2}\left[\frac{\partial^{2}}{\partial r_{i}^{2}}+\frac{1}{r_{i}} \frac{\partial}{\partial r_{i}}+\frac{1}{r_{i}^{2}} \frac{\partial^{2}}{\partial \theta_{i}^{2}}\right]+r_{i} \frac{\partial}{\partial r_{i}} \\
& -\frac{3 \alpha}{3 r_{2}^{2}-r_{1}^{2} e^{2 i\left(\theta_{2}-\theta_{1}\right)}}\left[\frac{r_{2}^{2}-r_{1}^{2} e^{2 i\left(\theta_{2}-\theta_{1}\right)}}{r_{1}}\left(\frac{\partial}{\partial r_{1}}-\frac{i}{r_{1}} \frac{\partial}{\partial \theta_{1}}\right)\right. \\
& \left.+2 r_{2}\left(\frac{\partial}{\partial r_{2}}-\frac{i}{r_{2}} \frac{\partial}{\partial \theta_{2}}\right)\right]
\end{aligned}
$$

The full wave function is ofcourse,

$$
\psi=|X|^{\alpha} \exp \left(-(1 / 2) \sum_{i} r_{i}^{2}\right) \phi\left(r_{1}, r_{2}, \theta_{1}, \theta_{2}\right) \Phi_{C M}(R, \Theta)
$$

So far two asymptotic regions have been used to simplify the original eigenvalue equation:

(i) $r_{1}, r_{2} \rightarrow \infty$ such that $r_{1} / r_{2}$ is fixed and finite.

(ii) $r_{1}, r_{2} \rightarrow 0$ such that $r_{1} / r_{2}$ is fixed and finite.

and we arrived at the possibility of two different series for some wave functions. Clearly there are two more regions possible, namely,

(iii) $r_{1} \rightarrow 0$ such that $r_{2}$ is fixed or $r_{2} \rightarrow \infty$ such that $r_{1}$ is fixed,i.e., $r_{1} / r_{2} \rightarrow 0$.

(iv) $r_{2} \rightarrow 0$ such that $r_{1}$ is fixed or $r_{1} \rightarrow \infty$ such that $r_{2}$ is fixed,i.e., $r_{2} / r_{1} \rightarrow 0$. 
Notice that these types of regions become possible only for $N \geq 3$, when there are at least two relative coordinates. For two anyon problem there is a single relative coordinate and asymptotics for $\phi$ can be considered only as $r \rightarrow \infty$ or $r \rightarrow 0$ (regions (i) and (ii)). Since two anyons have no possibility of any finite $\mathrm{R}$ or scale parameter entering one has to use Taylor series, and hence the wave functions are necessarily polynomials. This leads to a linearly interpolating spectrum. This is true provided either that $\alpha$ dependence is assumed to be smooth or $\phi$ is $C^{\infty}$ function.

To focus, let us consider (iii). As $r_{1} / r_{2} \rightarrow 0$ the Hamiltonian becomes separable and $J_{i}=-i \frac{\partial}{\partial \theta_{i}}$ commute with the corresponding separated Hamiltonian. This is true under the assumption that the derivatives in the $\alpha$ dependent terms in the $H_{r e l}$ remain finite as $r_{1} / r_{2} \rightarrow 0$.

$$
H_{i}=-\frac{1}{2}\left[\frac{\partial^{2}}{\partial r_{i}^{2}}+\frac{1}{r_{i}} \frac{\partial}{\partial r_{i}}+\frac{1}{r_{i}^{2}} \frac{\partial^{2}}{\partial \theta_{i}^{2}}\right]+r_{i} \frac{\partial}{\partial r_{i}}-\frac{\beta_{i}}{r_{i}}\left(\frac{\partial}{\partial r_{i}}-\frac{i}{r_{i}} \frac{\partial}{\partial \theta_{i}}\right) ; i=1,2,
$$

where

$$
\beta_{1}=\alpha ; \quad \beta_{2}=2 \alpha
$$

and

$$
H=H_{1}+H_{2} \text {. }
$$

That $\beta_{1}=\alpha$ is obvious because by definition $r_{1}$ involves particles 1 and 2 . $\beta_{2}=2 \alpha$ follows from the fact that $r_{2}$ involves all the three particles, and in the asymptotic region particle 3 can only wind around particles 1 and 2 together, but not around any one of them seperately.

Because of this as $r_{1} / r_{2} \rightarrow 0$,

$$
\phi\left(r_{1}, r_{2}, \theta_{1}, \theta_{2}\right) \longrightarrow \phi_{1}\left(r_{1}, \theta_{1}\right) \phi_{2}\left(r_{2}, \theta_{2}\right),
$$


where,

$$
\phi_{i}\left(r_{i}, \theta_{i}\right)=\eta_{i}\left(r_{i}\right) \exp \left(i n_{i} \theta_{i}\right) ; n_{1}+n_{2}=j .
$$

Now consider $r_{1} / r_{2} \rightarrow 0$ as $r_{1} \rightarrow 0$ and $r_{2}$ finite and fixed. Let,

$$
\phi\left(r_{1}, r_{2}, \theta_{1}, \theta_{2}\right) \longrightarrow r_{1}^{\lambda} \exp \left(i n_{1} \theta_{1}\right) \phi_{2}\left(r_{2}, \theta_{2}\right)
$$

Since $\mathrm{H}$ is seperable, we may first compute $H_{1}$ acting on $\phi$. We have,

$$
H_{1} \phi=r_{1}^{\lambda} \exp \left(\operatorname{in}_{1} \theta_{1}\right) \phi_{2}\left(r_{2}, \theta_{2}\right)\left[\lambda-\frac{1}{2} \frac{\left(\lambda^{2}-n_{1}^{2}+2 \alpha\left(n_{1}+\lambda\right)\right)}{r_{1}^{2}}\right] .
$$

Because $r_{1} \rightarrow 0$ limit has to be finite (leading power is already taken out) we get,

$$
\lambda^{2}-n_{1}^{2}+2 \alpha\left(n_{1}+\lambda\right)=0 \Longrightarrow \lambda=-\alpha \pm\left|n_{1}-\alpha\right|
$$

Now in the same limit, we have $|X|^{\alpha} \rightarrow r_{1}^{\alpha} r_{2}^{2 \alpha}$ and therefore the total power of $r_{1}$ becomes $\pm\left|n_{1}-\alpha\right|$. Since $\phi$ has to vanish as $r_{1} \rightarrow 0$ ( $r_{2}$ fixed) we have to choose the positive sign.

This also matches with the two anyon wave function in relative coordinates which vanishes as $r^{|n-\alpha|}$. Thus choosing the positive sign,

$$
\lambda=-\alpha+\left|n_{1}-\alpha\right|
$$

we have

$$
\begin{aligned}
\left(H_{1}+H_{2}\right) \phi & =\left[\left(H_{2}+\lambda\right) \phi_{2}\right] r_{1}^{\lambda} \exp \left(i n_{1} \theta_{1}\right) \\
& =(E-2-3 \alpha) \phi_{2} r_{1}^{\lambda} \exp \left(i n_{1} \theta_{1}\right)
\end{aligned}
$$

Thus $\phi_{2}$ satisfies the eigenvalue equation

$$
H_{2} \phi_{2}=(E-2-3 \alpha-\lambda) \phi_{2}=\left(E-2-2 \alpha-\left|n_{1}-\alpha\right|\right) \phi_{2}=\mathcal{E} \phi_{2} .
$$


$H_{2}$ is once again the "two anyon Hamiltonian". There is one crucial difference; since our equations are valid only asymptotically $r_{1} / r_{2} \rightarrow 0$ we have to maintain $r_{2}$ nonzero. Let us say that $r_{2} \geq R_{0}$, where $R_{0}$ is some new parameter.

With the definitions

$$
\begin{aligned}
\mu & =\frac{1}{2}\left[-2 \alpha+\left|n_{2}-2 \alpha\right|\right] \\
b & =1+\left|n_{2}-2 \alpha\right| \\
a & =-\frac{1}{2}\left[\mathcal{E}+2 \alpha-\left|n_{2}-2 \alpha\right|\right] \\
& =-\frac{1}{2}\left[E-2-\left|n_{1}-\alpha\right|-\left|n_{2}-2 \alpha\right|\right] \\
x & =r_{2}^{2} \\
R & =R_{0}^{2} \\
\phi_{2}\left(r_{2}, \theta_{2}\right) & =x^{\mu} \exp \left(\operatorname{in}_{2} \theta_{2}\right) u(x)
\end{aligned}
$$

the eigenvalue equation for $\phi_{2}$ becomes the CFE for $\mathrm{u}(\mathrm{x})$ :

$$
\begin{gathered}
{\left[x \frac{d^{2}}{d x^{2}}+(b-x) \frac{d}{d x}-a\right] u(x)=0,} \\
R \leq x<\infty .
\end{gathered}
$$

The general solution is given by,

$$
u(x)=C_{1} M(a, b, x)+C_{2} U(a, b, x),
$$

where $\mathrm{M}$ and $\mathrm{U}$ are in standard notation [14] and $C_{1}$ and $C_{2}$ are constants.

For $C_{1}$ we have two possibilities, namely it is zero or non zero. If it is non zero then normalizability as $x \rightarrow \infty$ requires a to be minus a non negative integer. This is precisely the case when the $U$ solution ceases to be 
an independent solution. In other words, if $C_{1}$ is non zero then we really have only one solution which is a finite order polynomial. This solution is also well behaved as $\mathrm{x}$ goes to zero and therefore we may take $\mathrm{R}$ to be zero. The energy is read off by putting the value of a in the definitions given above. We see that the energy eigenvalue in this case is linear in $\alpha$. The known exact solutions are covered by this case.

If $C_{1}$ is zero then $C_{2}$ is non zero and we have only the $\mathrm{U}$ solution. $\mathrm{U}$ is well behaved for large $\mathrm{x}$ and thus we do not get any condition on a from the demand of normalizability. However $\mathrm{U}$ is divergent as $x \rightarrow 0$ and we have to keep $\mathrm{R}$ strictly positive.

The only way E can get quantised now is by putting a boundary condition at $x=R$. Hermiticity analysis implies $\left.U\right|_{R}=0$ or $d U /\left.d x\right|_{R}=0$. But

$$
\frac{d U}{d x}=-a U(a+1, b+1, x)
$$

Because a is not a non positive integer (else we are back to the previous case ) essentially we get the conditions

$$
U(a, b, x)=0
$$

or

$$
U(a+1, b+1, x)=0 .
$$

Now $\mathrm{U}(\mathrm{a}, \mathrm{b}, \mathrm{x})$ has real positive zeros only if [14

$$
a<0 \quad \text { and } \quad 1+a-b<0 .
$$

In the present case $b=1+\left|n_{2}-2 \alpha\right|$ and

$$
1+a-b=a-\left|n_{2}-2 \alpha\right|
$$


Zeros of $\mathrm{U}$ exist only if,

$$
\begin{aligned}
a<0 \quad \text { and } & a<\left|n_{2}-2 \alpha\right| \quad(\text { if } U=0) \\
& \text { or } \\
a+1<0 \quad \text { and } & a<\left|n_{2}-2 \alpha\right| \quad\left(\text { if } U^{\prime}=0\right),
\end{aligned}
$$

that is for $a<0$ or $a<-1$ respectively. In the limit $\alpha \rightarrow 0$ we know that all solutions are finite polynomials. In effect then $R \rightarrow 0$ must hold as $\alpha \rightarrow 0$, i.e., a must be a negative integer. The Bosonic ground state has energy $2 \hbar \omega$. To admit this possibility we must allow for $a$ to be zero. We will therefore choose $U(a, b, R)=0$ as our boundary condition. Since for $a<0$ (a not an integer) $\mathrm{U}$ has in general more than one zero we will stipulate $R$ to be the smallest zero of $\mathrm{U}$ (say). This then determines uniquely $a$ and hence $\mathrm{E}$ as a function of $\mathrm{R}$ which is still an unknown parameter.

It may seem that the above boundary condition is very restrictive. It is not. For suppose we knew $E=E(\alpha)$, then $\mathrm{U}$ has zeroes and hence the smallest zero. Therefore for all $\alpha$, we can always find an $\mathrm{R}$ such that, $U(a(\alpha), b, R)=0$. But now clearly $\mathrm{R}$ depends on $\alpha$ and the asymptotic quantum numbers $n_{1}, n_{2}$. Thus $a\left(\alpha, n_{1}, n_{2}\right)$ determines $R=R\left(\alpha, n_{1}, n_{2}\right)$. Conversely, if we could determine $R=R\left(\alpha, n_{1}, n_{2}\right)$ by some arguement then $\mathrm{U}=0$ will determine $E\left(\alpha, n_{1}, n_{2}\right)$.

The boundary condition can thus be understood as stipulating that there exists functions $a(\alpha), R(\alpha)$ and $b(\alpha)$ which is explicitly known, such that $U(a, b, R)=0$, i.e., in the space parametrised by variables $\mathrm{E}$, $\mathrm{b}$ and $\mathrm{R}$ with $2 \leq E<\infty, b=1+\left|n_{2}-2 \alpha\right|, 0<R<\infty$ there exist curves along which $\mathrm{U}=0$. Determining these curves is equivalent to getting the spectrum. The 
differential statement of $U=0$ along a curve is of course $\frac{d U}{d \alpha}=0$ which can be written as:

$$
\frac{\partial U}{\partial a} \frac{d a}{d \alpha}+\frac{\partial U}{\partial b} \frac{d b}{d \alpha}+\frac{\partial U}{\partial R} \frac{d R}{d \alpha}=0
$$

Meanwhile we have tested the $U=0$ criterian in the following way. We choose a particular state at the fermionic or the bosonic end. Use the numerically determined eigenvalue to get $a(\alpha)$ and determine the zero of the $\mathrm{U}$ function numerically. Thus we get a $\mathrm{R}(\alpha)$. We fit a form to thus determined $\mathrm{R}$ and the compute $\mathrm{E}$ at arbitrary values of $\alpha$. This still has predictive power in that once we postulate a form for $\mathrm{R}(\alpha)$ we can determine its parameters for small values of $\alpha$ where perturbation theory can be used. We then have a prediction for all values of $\alpha$ in the range $[0,1]$. In particular we have carried out this exercise for the state which interpolates to the fermionic ground state and the corresponding bosonic state with $\mathrm{j}=2$ which is supersymmetric [8] to the fermionic ground state. The results are given in the figure 1 for the energy and figure 2 for the corresponding $\mathrm{R}$.

In the end we briefly mention another method of analysing solutions in the three anyon problem using the Fourier expansion of the wavefunction $\phi$ in eq.(31). Note that $\phi$ may be written as,

$\phi_{j}\left(r_{1}, r_{2}, \theta_{1}, \theta_{2}\right)=\sum_{n_{1}, n_{2}} e^{i n_{1} \theta_{1}} e^{i n_{2} \theta_{2}} \chi_{n_{1}, n_{2}}\left(r_{1}, r_{2}\right)=\sum_{n} e^{i j \frac{\left(\theta_{1}+\theta_{2}\right)}{2}} e^{i n \frac{\left(\theta 1-\theta_{2}\right)}{2}} \chi_{j, n}\left(r_{1}, r_{2}\right)$

for a given $j$, where $j=n_{1}+n_{2}, n=n_{1}-n_{2}$. We can now use this representation to look at the solutions for a given $j$. The eigenvalue equation now becomes $H_{r e l} \phi_{j}=\mathcal{E} \phi_{\mid}$, where $H_{r e l}$ is the three-anyon Hamiltonian in 
relative coordinates and $\mathcal{E}=E-2-3 \alpha$ as before. Substituting for $\phi_{j}$ the eigenvalue equation takes the form,

$$
\begin{aligned}
& 3 x_{2}\left[4 x_{1} \frac{\partial^{2}}{\partial x_{1}^{2}}+4\left(1+\alpha-x_{1}\right) \frac{\partial}{\partial x_{1}}-\frac{(j+n)(j+n-4 \alpha)}{4 x_{1}}\right. \\
+ & \left.4 x_{2} \frac{\partial^{2}}{\partial x_{2}^{2}}+4\left(1+2 \alpha-x_{2}\right) \frac{\partial}{\partial x_{2}}-\frac{(j-n)(j-n-8 \alpha)}{4 x_{2}}+2 \mathcal{E}\right] \chi_{j, n} \\
= & x_{1}\left[4 x_{1} \frac{\partial^{2}}{\partial x_{1}^{2}}+4\left(1+3 \alpha-x_{1}\right) \frac{\partial}{\partial x_{1}}-\frac{(j+n+4)(j+n+4-12 \alpha)}{4 x_{1}}\right. \\
+ & \left.4 x_{2} \frac{\partial^{2}}{\partial x_{2}^{2}}+4\left(1-x_{2}\right) \frac{\partial}{\partial x_{2}}-\frac{(j-n-4)^{2}}{4 x_{2}}+2 \mathcal{E}\right] \chi_{j, n+4},
\end{aligned}
$$

where $x_{i}=r_{i}^{2}$. This equation is exact. These infinite set of coupled equations relate Fourier modes differing by 4 , i.e., if $\chi_{0}, \chi_{1}, \chi_{2}, \chi_{3}$ are know then $\chi_{4 k}, \chi_{4 k+1}, \chi_{4 k+2}, \chi_{4 k+3}$ get determined in terms of $\chi_{0}, \ldots, \chi_{3}$ etc. However there is no relation among $\chi_{0}, \chi_{1}, \chi_{2}, \chi_{3}$ themselves. In a sense these four functions will give four independent solutions of the eigenvalue equations. We can then deal with a given "tower" separately and independently and this is true for every given $\mathrm{j}$. Let us concentrate on one tower. Now three distinct cases arise naturally:

(a) Only one member of the tower is nonzero, ie., $\chi_{j, n}=\chi_{j, m} \delta_{n, m}$.

(b) Only a finite number of $\chi_{j, n}$ 's are nonzero, ie., $\chi_{j, n}=0 \quad \forall n \geq n_{1} \quad$ and $\quad \forall n \leq n_{2}$ with $n_{2}<n_{1}$.

(c) Infinitely many $\chi_{j, n}$ 's are nonzero. This gives raise to the following cases:

$$
\begin{aligned}
& \chi_{j, n}=0 \forall n \geq n_{1} \\
& \chi_{j, n}=0 \forall n \leq n_{2} \\
& \chi_{j, n} \neq 0 \forall n .
\end{aligned}
$$


Case (a) is simple to analyse and it reproduces the known exact solutions. In the exact eigenvalue equation relating $\chi_{j, n}$ and $\chi_{j, n+4}$ given above we set lhs to zero which gives an equation for the $\chi_{j, n}$. But the rhs also gives rise to another equation for the same $\chi_{j, n}$ when the coupling to $\chi_{j, n-4}$ is taken into account. However the form of equations suggests that the two equations are seperable but must be consistently solved. The consistency conditions immediately yield the linear exact solutions which are already outlined previously. Cases (b) and (c) seem quite complicated and nonlinearly interpolating states must be in one of these cases. Consider the normalisation condition on the full wavefunction,

$$
\left\|\psi_{j}\right\|^{2}=\sum_{n} \int_{0}^{\infty} d x_{1} d x_{2}|X|^{2 \alpha}\left|\chi_{j, n}\right|^{2} e^{-x_{1}+x_{2}} \equiv \sum_{n} C_{n}
$$

If $\psi_{j}$ is given by case (b) then there are only a finite number of terms in the norm and if $C_{n}$ 's are finite then $\psi_{j}$ is normalisable. Thus the quantisation condition for the nonlinear states must arise by the demand that $C_{n}$ must be finite. For the case (c) even if all $C_{n}$ 's are finite we may still get the wavefunction to be infinite because the sum above may not converge and then the quantisation condition would be the convergence of $\sum_{n} C_{n}$.

We have not at present analysed this scenario in detail. But it does appear that the case (b) is ruled out in which case the exact solutions come from case (a) and the nonlinear solutions arise from case (c). 


\section{Summary and Discussion}

We have in this paper, analysed the problem of three anyons confined in an oscillator potential. The energy spectrum of three anyons generically contains two types of interpolations as a function of the statistical parameter $\alpha$. The first type are the linearly interpoating eigenvalues with $\Delta E=E(\alpha=$ $1)-E(\alpha=0)=3$. These solutions are easily generalised to N-anyon case where $\Delta E=N(N-1) / 2, N \geq 2$. The second set consists of solutions for eigenvalues which are in general non-linear functions of $\alpha$. In this paper we have outlined an analytical method of obtaining such solutions. We exploit the known asymptotics to arrive at these solutions. Since the differential equation is valid in all regions it is sufficient to specify the wavefunction asymptotically to obtain the exact eigenvalues.

For $\mathrm{N}=3$, there are two relative coordinates whose magnitudes are given by $r_{1}, r_{2}$. The asymptotic regions are then defined by,

- (a) $r_{1}, r_{2} \rightarrow \infty$.

- (b) $r_{1}, r_{2} \rightarrow 0$.

- (c) $r_{1} \rightarrow 0, \quad r_{2}$ fixed; or $\quad r_{2} \rightarrow \infty, r_{1}$ fixed.

- (d) $r_{2} \rightarrow 0, \quad r_{1}$ fixed; or $\quad r_{1} \rightarrow \infty, r_{2}$ fixed.

Asymptotic region (a) immediately yields the Gaussian which controls the behaviour of the wavefunction at infinity and region (b) yields the Laughlin$\mathrm{Wu}$ factor which regulates the wave function as two anyons approach each 
other. The asymptotic region (c) (or (d)) is used to obtain the nonlinearly interpolating solutions.

There is a caveat to note: There may be exact eigenfunctions which do not satisfy the asymptotic equation (33). In this case our analysis can at best be expected to give an approximation to the exact eigenvalues. In the absense of a complete rigorous proof precluding this possiblity we have to note it as an open one.

A few additional points are worth noting.

i) We have chosen $R$ to be the smallest zero of $U$ without any justification. For low lying eigenvalues the $U$ function has only one zero so there is no ambiguity. For higher states though more zeros are possible and their implications are to be explored and elaborated.

ii) Some qualitative properties such as a tower of evenly spaced eigenvalues for every non linearly interpolating eigenvalue [9] and "supersymmetry" for a class of eigenstates [ 8 have been noted in the literature cited. Whether and how these fit into our appraach is to be seen.

\section{Acknowledgements}

We thank G.Baskaran, Diptiman Sen and P.N.Srikanth for valuable discussions. We also thank Patricia Monger for the Super Mongo software, which helped us in figuring out the zeros of Confluent Hypergeometric Functions. 


\section{References}

[1] J M Leinaas and J Myrheim, Nuovo Cimento, 37B, (1977) 1.

[2] F Wilczek, Phys. Rev. Lett.48, 1144 (1982); ibid, 49, (1982)957;

F Wilczek and A Zee, Phys. Rev. Lett. 51, (1983)2250.

For some recent reviews, see R McKenzie and F Wilczek, Int. Jour. Mod.

Phys, A12, (1988)2827;

G S Canright and M D Johnson, Comments on Condensed Matter Physics, 15, (1990)77.

[3] Yong-Shi Wu, Int.J.Mod.Phys.B5(1991)1649.

[4] G.Date, T.R.Govindarajan, P. Sankaran and R.Shankar, Commun.Math.Phys. 132, 293 (1990) and references therein;

[5] B Halperin, Phys. Rev. Lett.52, (1984)1583;

R B Laughlin in Quantum Hall Effect, eds. R E Prange and S M Girvin, Springer Verlag, New York 1987, p.233.

[6] R B Laughlin, Science, 242,(1988) 525 and references cited therein.

[7] C.Chou, Phys.Rev. D44, 2533 (1991);

A.P.Polychronakos, Phys.Lett. B264, 362 (1991);

G.Dunne, A.Lerda, S.Sciuto and C.A.Trugenberger, MIT Preprint CTP1978 (1991);

R.Basu, G.Date and M.V.N.Murthy, Phys.Rev.B46(1992)3139. 
[8] M.Sporre, J.J.M.Verbaarschot and I.Zahed, Phys.Rev.Lett. 67, (1991)1813;

M.V.N.Murthy, J.Law, M.Brack and R.K.Bhaduri, Phys.Rev.Lett. 67, (1991)1817;

M.Sporre, J.J.M.Verbaarschot and I.Zahed, Stony Brook Preprint SUNY -NTG - 91/40 (1991);

For a discussion of the Supersymmetry in the two and three anyon solutions see for example, Diptiman Sen, Bangalore Preprint, IISc-CTS91-9 and R.Chitra, C.Nagaraja Kumar and Diptiman Sen, Bangalore Preprint, IISc- CTS-91-8.

[9] Shuxi Li , R.K.Bhaduri and M.V.N.Murthy, Phys.Rev.B46(1992)1228;

R.Chitra and D.Sen, Bangalore Preprint,IISc-CTS-92-2.

[10] T.Awaji and M.Hotta, Tohoku Preprint (1991).

[11] J.McCabe and S.Ouvry, Phys.Lett. B260, (1991)113;

A.Comtet, J.McCabe and S.Ouvry, Phys. Lett. B260, (1991)372;

R.K.Bhaduri, R.S.Bhalerao, A.Khare, J.Law and M.V.N.Murthy, Phys. Rev. Lett. 66, (1991)523.

[12] A.Khare and J.McCabe, Phys.Lett. B269(1991)330

[13] M.V.N.Murthy, J.Law, R.K.Bhaduri and G.Date, McMaster University/IMSc Preprint (To appear in J.Phys.A (Mathematical and General)). 
[14] M.Abramowitz and I.A.Stegun, Handbook of Mathematical Functions, (Dover, NewYork, 1970)p.503;

L.J.Slater, Confluent Hypergeometric Functions(Cambridge Un.Press, Cambridge, 1960)p.102 


\section{Figure Captions}

Figure 1: Plot of the Fermionic ground state energy and the corresponding Bosonic $\mathrm{j}=2$ state as a function of $\alpha$ obtained by using the boundary condition at $\mathrm{R}$. The non-interacting Bosonic state is at $\alpha=0$ and the non-interacting Fermionic state is at $\alpha=1$ and energy $E=4$ in dimensionless units. The points shown along the Fermionic curve are taken from the numerical calculations [8].

Figure 2: Shows the values of $\mathrm{R}$ as a function of $\alpha$ used in computing the energies of the corresponding states. For the Fermionic ground state $R \approx 0.405 \alpha(1-\alpha)$ obtaied from numerical computation close to $\alpha=0$. The corresponding form for the Bosonic state is $R \approx \alpha^{0.6}$. 